\title{
Multiple Einsatzmöglichkeiten für eine individuellere Therapie
}

\author{
Bei Typ-2-Diabetes geht die Entwicklung hin zu einer möglichst \\ individuell angepassten Therapie. Dabei muss unter anderem \\ dem Erkrankungsstadium und den bereits eingetretenen Folge- \\ komplikationen Rechnung getragen werden.
}

Für den DPP-4-Hemmer Vildagliptin (Galvus $^{\circledR}$ ) zur oralen antidiabetischen Therapie hat es zuletzt einige Zulassungserweiterungen gegeben: u.a. auf Typ-2Diabetiker mit moderater oder schwerer Nierenschwäche und auf jene, die bei Insulintherapie in stabiler Dosis eine Therapie-Intensivierung benötigen.

Verschlechtert sich die Nierenfunktion eines Typ-2-Diabetikers von milder zu moderater Insuffizienz ist das Spektrum der einsetzbaren Antidiabetika schon deutlich eingeschränkt, so Prof. Matthias Blüher aus Leipzig. Die abnehmende Möglichkeit, Medikamente über die Niere auszuscheiden, macht diese Patienten empfindlicher in Bezug auf erwünschte und unerwünschte Wirkungen. Metfor- min als Arznei der ersten Wahl, sollte nach wie vor nur bis zur milden Niereninsuffizienz eingesetzt werden. Für Vildagliptin besteht der Vorteil, dass es auch ohne das First-line-Antidiabetikum einsetzbar ist. Zudem ist ab der moderaten Niereninsuffizienz nur eine Halbierung der Tagesdosis auf $50 \mathrm{mg}$ nötig, die bis hin zur terminalen Insuffizienz nicht weiter reduziert werden muss. Mangels Erfahrung bei Dialysepatienten sollte es hier allerdings mit Vorsicht eingesetzt werden, woran Blüher erinnert hat.

\section{Studien bei Nierenschwäche oder Insulinpflicht}

Dass Vildagliptin auch bei Typ-2-Diabetikern mit moderater bis schwerer Nie- reninsuffizienz (NI) effektiv und sicher wirkt, hat es etwa in einer Studie mit ca. 500 Patienten bewiesen [1]. Die Patienten erhielten zusätzlich zu ihrer bisherigen Therapie $50 \mathrm{mg} / \mathrm{d}$ Vildagliptin oder Placebo. Versus Placebo war der $\mathrm{HbA}_{1 c}$-Wert nach 24 Wochen mit dem Gliptin bei Patienten mit moderater NI um signifikante $0,48 \%$-Punkte verringert, bei schwerer NI um 0,57\%-Punkte. Eine Zunahme von unerwünschten Effekten mit dem Gliptin war nicht zu beobachten.

In der Kombination mit Insulin hat sich Vildagliptin in einer Studie mit etwa 450 Typ-2-Diabetikern bewährt [2]. Sie erhielten über 24 Wochen zweimal täglich $50 \mathrm{mg}$ Vildagliptin oder Placebo zusätzlich zu ihrer Therapie aus Basalinsulin in stabiler Dosis mit oder ohne Metformin. Versus Placebo sank der $\mathrm{HbA}_{1 \mathrm{c}}{ }^{-}$ Wert um 0,7\%-Punkte deutlich ab, und zwar ohne die Hypoglykämiehäufigkeit oder das Körpergewicht signifikant zu steigern.

Sarah Louise Pampel

1. Lukashevich V et al. Diabetes Obes Metab 2011;13:947-54.

2. Kothny W et al. Diabetes Obes Metab 2013:15:252-7 und Novartis data on file.

Quelle : Galvus Gold 2013, 26.-27.4.2013 in Rom, Veranstalter: Novartis

\section{Prandialer GLP-1-Agonist ist guter Partner des Basalinsulins}

\begin{abstract}
Seit März 2013 steht mit Lixisenatid ein neuer prandialer GLP-1-Agonist für die Diabetestherapie zur Verfügung, der in klinischen Studien v.a. in Kombination mit dem Basalinsulin Glargin untersucht wurde.
\end{abstract}

nerte Hanefeld. Diese seien ein eigenständiger kardiovaskulärer Risikofaktor. Behandelt werden können sie mit kurz wirksamen Insulinen, die aber kombiniert mit Basalinsulin Gewichtszunahme und Hypoglykämie fördern. Alternativ gibt es kurz wirksame GLP-1-Agonisten wie Lixisenatid $\left(\right.$ Lyxumia $\left.^{\mathbb{R}}\right)$, das seit 15.3.2013 in Deutschland erhältlich ist.

Solange die Inselzellen noch nicht völlig erschöpft sind, sind GLP-1-Agonisten sinnvoller, sicherer und bequemer, so
Prof. Thomas Forst, Mainz. Denn sie adressieren die Dysfunktion der Alfa- und Betazellen, verzögern die Magenentleerung und wirken erst nach Glukosebelastung. Die Folge: antidiabetischer Effekt bei sehr geringem Hypoglykämierisiko und Gewichtsverlust von ca. $2 \mathrm{~kg}$ in $1 \mathrm{Jahr}$ mit Lixisenatid, wobei v.a. das kardiovaskulär bedenkliche viszerale Fett schwinde..

Lixisenatid ist der erste prandiale GLP1-Agonist zur einmal täglichen Anwendung, so Dr. Milan Novakovic, Berlin, von Sanofi. Empfohlen wird eine einmalige Titrierung bis zur Erhaltungsdosis von $20 \mu \mathrm{g} / \mathrm{d}$, s.c. vor der Hauptmahlzeit. In klinischen Studien wurde die Substanz bei 2600 Patienten getestet, oft kombiniert mit Glargin. In den Studien GetGoal Duo-1 und GetGoal-L wurde mit Lixisenatid eine signifikante $\mathrm{HbA}_{\mathrm{lc}}$-Senkung erreicht, die um $0,3-0,4 \%$ stärker war als mit Placebo. Dr. med. Dirk Einecke

Quelle: Einführungspressekonferenz am 19.3.2013 in Neu Isenburg, Veranstalter: Sanofi 